\title{
The effect of bilirubin on long-term mortality in patients with chronic total coronary occlusion
}

\author{
Cetin $\mathrm{M}^{1}$, Durakoglugil $\mathrm{ME}^{1}$, Erdogan $\mathrm{T}^{1}$, Ozer $\mathrm{S}^{2}$, Turan $\mathrm{OE}^{3}$, Yilmaz $\mathrm{AS}^{1}$, Akdogan $\mathrm{E}^{4}$ \\ Recep Tayyip Erdogan University, Faculty of Medicine, Department of Cardiology, Rize, Turkey. \\ emredur@hotmail.com
}

\begin{abstract}
AIM: We intended to investigate the association of bilirubin with total mortality in patients with chronic total coronary occlusion (СTO).

METHODS: We included 172 patients who underwent coronary angiography due to stable angina pectoris and had CTO. We checked the viability of patients after 9 years of follow-up.

RESULTS: Direct bilirubin levels were significantly lower in the non-viable group. We revealed age (OR

$=1.045,95 \%$ C.I: $1.009-1.083 ; p=0.015)$ and direct bilirubin concentrations $(\mathrm{OR}=0.029,95 \%$ C.I:

$0.002-0.435 ; p=0.029$ ) as independent predictors of mortality. Direct bilirubin value of $>0.2 \mathrm{mg} / \mathrm{dL}$ was associated with decreased mortality with a sensitivity of $85 \%$, and a specificity of $46 \%$.

CONCLUSION: Serum direct bilirubin concentrations independently predict total mortality in patients with chronic total occlusion over 9 years of follow-up (Tab. 1, Fig. 2, Ref. 23). Text in PDF www.elis.sk.

KEY WORDS: bilirubin, chronic total occlusion, long-term mortality.
\end{abstract}

\section{Introduction}

Bilirubin, the end product of the catabolism of heme, is an important marker of liver function and bile excretion. Clinical and experimental studies identified bilirubin as a natural anti-oxidant that specifically inhibits lipid peroxidation (1). Serum bilirubin concentrations are inversely related with the risk of premature coronary artery disease (CAD), diabetes mellitus (DM), hypertension, and metabolic syndrome (2-5). Moreover, higher bilirubin levels are associated with lower rate of cardiovascular events such as myocardial infarction, congestive heart failure, ischemic stroke, peripheral artery disease and mortality (6-9).

We previously demonstrated a significant association between bilirubin concentrations and coronary collateral development (10). Although bilirubin is related with several cardiovascular endpoints, there is no information in the current literature regarding the influence of bilirubin on all-cause mortality in patients with chronic total occlusion (CTO). Therefore, we intended to investigate this association.

${ }^{1}$ Recep Tayyip Erdoğan University, Faculty of Medicine, Department of Cardiology, Rize, Turkey, ${ }^{2}$ Recep Tayyip Erdogan University, Education and Research Hospital, Department of Cardiology, Rize, Turkey, ${ }^{3}$ Karadeniz Technical University, Faculty of Medicine Department of Cardiology, Trabzon, Turkey, and ${ }^{4}$ Recep Tayyip Erdoğan University, Faculty of Medicine, Department of Hematology, Rize, Turkey

Address for correspondence: M.E. Durakoğlugil, Dr, Recep Tayyip Erdoğan University, School of Medicine, Department of Cardiology, Islampasa Mah., 53100, Rize, Turkey.

Phone: +90.464.2123009, Fax: +90.464.2123015

\section{Methods}

Our study is retrospective and observational. We included 172 consecutive patients who underwent coronary angiography due to stable angina pectoris and had chronic total coronary occlusion between May 2009 and December 2010. The study was performed in accordance with the principles stated in the Declaration of Helsinki. The local ethics committee approved the study protocol. Patients were checked for viability using hospital and social security database, and telephone contact.

Clinical characteristics, involving the history and physical examination of each patient, were gathered at the time of cardiac catheterization and stored in the database of coronary angiography laboratory at our institution.

Standard selective coronary angiography with at least 4 views of the left coronary system and 2 views of the right coronary artery was performed in all patients using the Judkins technique. Total coronary artery occlusion was defined as $100 \%$ luminal diameter stenosis without a discernable lumen and the absence of anterograde flow. CTO was defined as total coronary artery occlusion of $\geq 3$ months in duration.

Patients who had CTO in at least one major coronary artery were included in the current study. The coronary angiograms were reevaluated for collateral development by two experienced interventional cardiologists who were totally blinded to the study. The grade of coronary collateral development was determined according to the Cohen-Rentrop method (11). Patients with grade $0-1$ collateral development were regarded as poor collateral group and patients with grade 2-3 collateral development were regarded as good collateral group (12). Collateral grading 
was performed for the vessel with CTO. If a patient had more than one vessel with CTO and collateral development, collateral grading was defined according to the vessel that had better collateral development.

Patients with symptomatic peripheral vascular disease, cerebrovascular disease, prior PCI and/ or CABG, non-ischemic dilated cardiomyopathy, evidence of ongoing infection or inflammation, hepatic or cholestatic disease, recent acute coronary syndrome either with or without ST-segment elevation (one month within enrollment), hematological disorders and known malignancy were excluded from the study.

Blood samples were drawn by venipuncture to perform routine blood chemistry analyses after fasting for at least 8 hours before coronary angiography. Fasting blood glucose, serum creatinine, serum bilirubin, total cholesterol, high-density lipoprotein (HDL) cholesterol, low-density lipoprotein (LDL) cholesterol, and triglyceride levels were recorded. Glucose, creatinine, and lipid profile were determined by standard methods.

Statistical analysis

Continuous variables were presented as mean \pm standard deviation, and categorical variables were presented as percentages. The variables were investigated using visual (histograms, probability plots) and analytical methods (Kolmogorov-Smirnov/ShapiroWilk's test) to determine the distribution of data. Student t test was used to compare normally distributed parameters among the mortality groups. The Mann-Whitney U-test tests were conducted to compare not normally distributed parameters among the mortality groups. Cross tabulations were used for comparison of the proportions of patients with categorical variables. The Chi-square or Fisher's exact test (when chi-square test assumptions do not hold due to low expected cell counts) was used to compare different groups. For multivariate analysis, possible factors identified with the univariate analyses were further entered into the logistic regression analysis to determine independent predictors of mortality. Hosmer-Lemeshow goodness of fit statistics was used to assess model fit. A $5 \%$ type-I error level was used to infer statistical significance. Statistical analyses were performed using the SPSS software (Version 23.0, SPSS, Inc., Chicago, IL).

\section{Results}

\section{Demographic parameters}

Among 172 patients 55 (31.9\%, 7 female, 48 male) subjects died during follow-up. We formed two groups according to mortality. Patients who died were older (61.34 \pm 9.63 vs $66.76 \pm 9.33$ years; $p=0.001)$. The other demographic characteristics were similar between groups.

\section{Laboratory values}

Laboratory values were not different except direct bilirubin concentrations. Direct bilirubin levels were significantly lower in the non-viable group $(0.28 \pm 0.15$ vs $0.23 \pm 0.11 \mathrm{mg} / \mathrm{dL} ; \mathrm{p}=$ $0.031)$. There was a non-significant trend for increased platelet count and creatinine in the mortal group (Tab. 1).
Tab. 1. Demographic, biochemical, and angiographic parameters of study group.

\begin{tabular}{|c|c|c|c|}
\hline & & & \\
\hline & Alive $(n=117)$ & Dead $(n=55)$ & $\mathrm{p}$ \\
\hline \multicolumn{4}{|l|}{ Demographic parameters } \\
\hline$\overline{\text { Age (years) }}$ & $61.34 \pm 9.63$ & $66.76 \pm 9.33$ & 0.001 \\
\hline Gender (female \%) & $21(17.9 \%)$ & $7(12.7 \%)$ & 0.387 \\
\hline Diabetes & $47(40.2 \%)$ & $17(31.5 \%)$ & 0.275 \\
\hline Hypertension & $59(57.8 \%)$ & $31(64.6 \%)$ & 0.432 \\
\hline Dyslipidemia & $85(72.6 \%)$ & $43(78.2 \%)$ & 0.438 \\
\hline Smoking & $48(47.1 \%)$ & $27(56.3 \%)$ & 0.294 \\
\hline LV EF & $49.94 \pm 13.68$ & $46.58 \pm 11.19$ & 0.186 \\
\hline \multicolumn{4}{|l|}{ Laboratory values } \\
\hline$\overline{\text { Glucose }(\mathrm{mg} / \mathrm{dL})}$ & $119.44 \pm 35.96$ & $123.40 \pm 53.25$ & 0.569 \\
\hline BUN (mg/dL) & $37.76 \pm 14.18$ & $42.07 \pm 17.26$ & 0.085 \\
\hline Creatinine $(\mathrm{mg} / \mathrm{dL})$ & $0.98 \pm 0.28$ & $1.09 \pm 0.56$ & 0.084 \\
\hline Total bilirubin (mg/dL) & $0.71 \pm 0.29$ & $0.67 \pm 0.25$ & 0.289 \\
\hline Direct bilirubin (mg/dL) & $0.28 \pm 0.15$ & $0.23 \pm 0.11$ & 0.031 \\
\hline Total Cholesterol (mg/dL) & $187.24 \pm 47.67$ & $198.71 \pm 47.94$ & 0.253 \\
\hline Triglycerides (mg/dL) & $148.27 \pm 82.77$ & $149.54 \pm 59.72$ & 0.922 \\
\hline $\mathrm{HDL}(\mathrm{mg} / \mathrm{dL})$ & $37.17 \pm 9.86$ & $36.17 \pm 8.28$ & 0.542 \\
\hline LDL (mg/dL) & $123.24 \pm 36.44$ & $128.27 \pm 32.91$ & 0.412 \\
\hline $\operatorname{WBC}\left(10^{3} / \mathrm{mm}^{3}\right)$ & $7.73 \pm 2.28$ & $7.91 \pm 2.24$ & 0.357 \\
\hline Lymphocytes $\left(10^{3} / \mathrm{mm}^{3}\right)$ & $2.16 \pm 0.82$ & $2.16 \pm 0.92$ & 0.635 \\
\hline Monocytes $\left(10^{3} / \mathrm{mm}^{3}\right)$ & $0.59 \pm 0.26$ & $0.65 \pm 0.31$ & 0.191 \\
\hline Hemoglobin (mg/dL) & $13.60 \pm 1.45$ & $13.47 \pm 1.46$ & 0.591 \\
\hline Platelets $\left(10^{3} / \mathrm{mm}^{3}\right)$ & $268.09 \pm 58.63$ & $291.33 \pm 105.06$ & 0.066 \\
\hline \multicolumn{4}{|l|}{ Angiographic parameters } \\
\hline \multicolumn{4}{|l|}{ Rentrop grade } \\
\hline Rentrop $0-1$ & $43(36.8 \%)$ & $21(38.2 \%)$ & \multirow{2}{*}{0.856} \\
\hline Rentrop 2-3 & $74(63.2 \%)$ & $34(61.8 \%)$ & \\
\hline \multicolumn{4}{|l|}{ Number of CTO } \\
\hline 1 & $75(79.8 \%)$ & $19(20.2 \%)$ & \multirow{2}{*}{0.243} \\
\hline 2 & $26(70.3 \%)$ & $11(29.7 \%)$ & \\
\hline \multicolumn{4}{|l|}{ Affected vessel } \\
\hline LAD & $47(50 \%)$ & $13(35.1 \%)$ & \multirow{3}{*}{0.003} \\
\hline RCA & $34(36.2 \%)$ & $24(64.9 \%)$ & \\
\hline $\mathrm{CX}$ & $13(13.8 \%)$ & $0(0 \%)$ & \\
\hline
\end{tabular}

$\overline{\text { CAD - Coronary artery disease, LDL - Low-density lipoprotein, HDL - High- }}$ density lipoprotein, CTO - chronic total coronary occlusion, LAD - left anterior descending, CX - circumflex, RCA - right coronary artery LV EF - Left ventricular ejection fraction

\section{Angiographic characteristics}

Subjects who died had higher involvement of the right coronary artery (RCA). Collateral flow grade or CTO count did not differ between groups. Admission medical treatment was not different.

Multivariate \& ROC Analysis

We revealed age $(\mathrm{OR}=1.045,95 \%$ C.I: $1.009-1.083 ; \mathrm{p}=$ $0.015)$ and direct bilirubin concentrations $(\mathrm{OR}=0.029,95 \%$ C.I: $0.002-0.435 ; p=0.029)$ as independent predictors of mortality. AUC values of direct bilirubin were 0.349 . Direct bilirubin value of $>0.2 \mathrm{mg} / \mathrm{dL}$ was associated with decreased mortality having high sensitivity ( $85 \%$ ), but low specificity (46\%). Kaplan-Meier survival curve is presented in Figure 1.

\section{Discussion}

We discovered that age and direct bilirubin concentrations were predictors of all-cause mortality independent of good collateral 


\section{0-863}

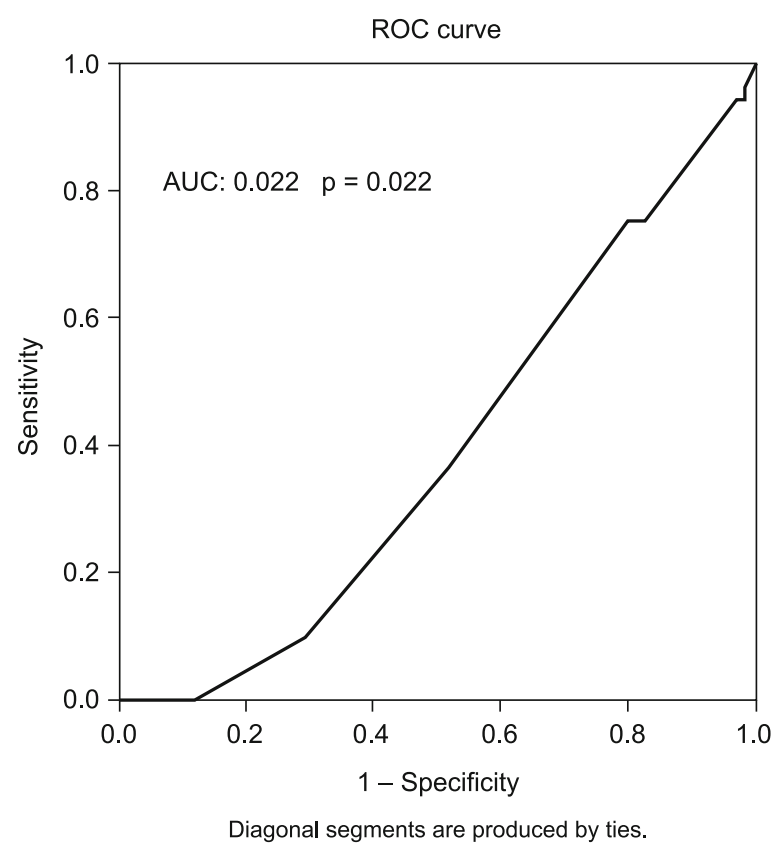

Fig. 1. ROC curve analysis of age and direct bilirubin for detecting total mortality.

development in patients with CTO during 9 years follow-up. As far as we know, our study is the first to demonstrate the relationship of bilirubin in this specific group with long-term observation.

As we mentioned previously, serum direct bilirubin is inversely related to both atherosclerotic risk factors, and cardiovascular morbidity and mortality (13). Although the exact pathophysiological process is not clear, the strong anti-oxidant activity of bilirubin is widely accepted as the major mechanism $(14,15)$. Oxidative stress plays an important role in atherosclerosis (16). Therefore, higher bilirubin concentrations may be associated with decreased oxidization of lipids and lipoproteins, which in return diminishes atherogenic plaque formation. Data suggests that a relationship exists between bilirubin and peripheral artery disease and carotid intima-media thickness $(8,17,18)$. Turfan and coworkers revealed that serum bilirubin levels were independently and inversely associated with high Syntax score in patients with stable CAD (19). Similarly, Chang et al. demonstrated that bilirubin is related with coronary artery disease complexity and severity assessed by SYNTAX score and 1-year major adverse cardiovascular events in patients with stable angina pectoris undergoing revascularization (20). Thus, low serum bilirubin seems to be associated with the severity of CAD besides being a risk factor for enhanced atherogenesis. We think that the same mechanism may also have a role in decreased long-term mortality as in our study.

Inflammation is also closely related to atherosclerosis. Akboga and coworkers demonstrated that bilirubin correlated inversely with inflammatory mediators including CRP (21). We may hypothesize that bilirubin may decrease plaque progression and vulnerability by reducing LDL oxidation, with concomitant decreases in local and systemic inflammation.

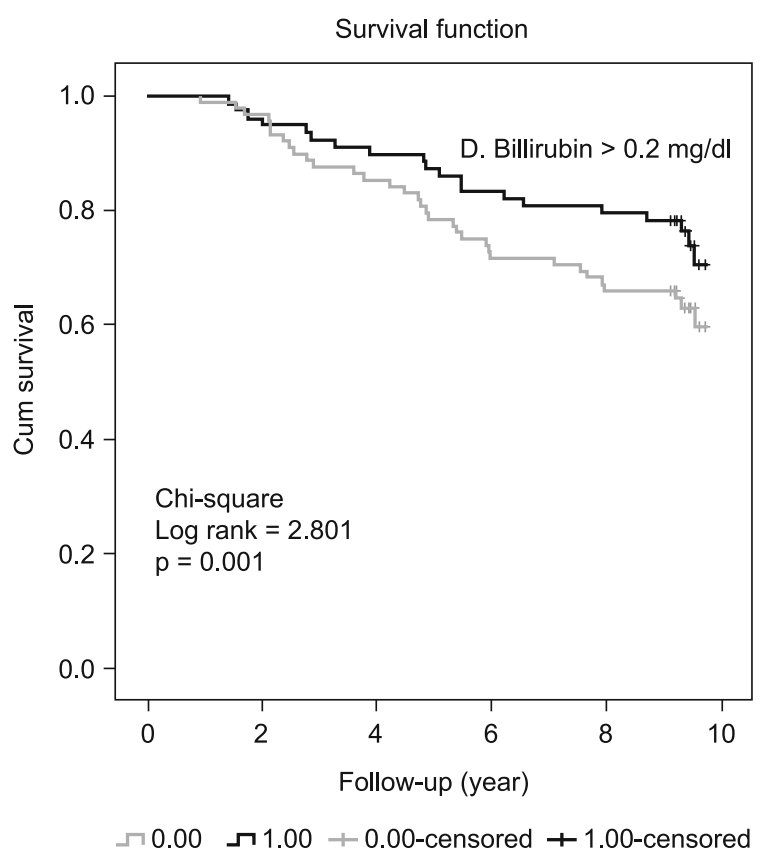

Fig. 2. Kaplan-Meier Survival graph of patients according to direct bilirubin $>0.2 \mathrm{mg} / \mathrm{dL}$ or $\leq 0.2 \mathrm{mg} / \mathrm{dL}$.

Good collateral development is associated with increased survival $(22,23)$. Interestingly we found that coronary collateral development grade, either poor or good, was not associated with mortality. We think that very long follow-up duration in this specific population may have caused this contradictory result.

Our study has several limitations. Mainly the study group is relatively small. Moreover, we do not have the data regarding the long-term medications, drug adherence, and revascularization during follow-up. We do not have any information regarding other adverse events. However, we investigated death, a hard end-point, which is the strongest part of our study.

\section{Conclusion}

Serum direct bilirubin concentration independently predicts total mortality in patients with chronic total occlusion over 9 years of follow-up.

\section{References}

1. Lin JP, Vitek L, Schwertner HA. Serum bilirubin and genes controlling bilirubin concentrations as biomarkers for cardiovascular disease. Clin Chem 2010; 56 (10): 1535-1543.

2. Hopkins PN, Wu LL, Hunt SC, James BC, Vincent GM, Williams RR. Higher serum bilirubin is associated with decreased risk for early familial coronary artery disease. Arterioscler Thromb Vasc Biol 1996; 16 (2): 250-255.

3. Han SS, Na KY, Chae DW, Kim YS, Kim S, Chin HJ. High serum bilirubin is associated with the reduced risk of diabetes mellitus and diabetic nephropathy. The Tohoku journal of experimental medicine 2010; 221 (2): 133-140. 
4. Chin HJ, Song YR, Kim HS et al. The bilirubin level is negatively correlated with the incidence of hypertension in normotensive Korean population. J Korean Med Sci 2009; 24 Suppl S50-56.

5. Giral P, Ratziu V, Couvert $P$ et al. Plasma bilirubin and gammaglutamyltransferase activity are inversely related in dyslipidemic patients with metabolic syndrome: relevance to oxidative stress. Atherosclerosis 2010; 210 (2): 607-613.

6. Marconi VC, Duncan MS, So-Armah K et al. Bilirubin Is Inversely Associated With Cardiovascular Disease Among HIV-Positive and HIVNegative Individuals in VACS (Veterans Aging Cohort Study). J Am Heart Assoc 2018; 7 (10)

7. Ong KL, Allison MA, Cheung BM, Wu BJ, Barter PJ, Rye KA. The relationship between total bilirubin levels and total mortality in older adults: the United States National Health and Nutrition Examination Survey (NHANES) 1999-2004. PloS one 2014; 9 (4): e94479.

8. Perlstein TS, Pande RL, Beckman JA, Creager MA. Serum total bilirubin level and prevalent lower-extremity peripheral arterial disease: National Health and Nutrition Examination Survey (NHANES) 1999 to 2004. Arterioscler Thromb Vasc Biol 2008; 28 (1): 166-172.

9. Perlstein TS, Pande RL, Creager MA, Weuve J, Beckman JA. Serum total bilirubin level, prevalent stroke, and stroke outcomes: NHANES 1999-2004. Am J Med 2008; 121 (9): 781-788 e781.

10. Erdogan T, Cicek Y, Kocaman SA et al. Increased serum bilirubin level is related to good collateral development in patients with chronic total coronary occlusion. Intern Med 2012; 51 (3): 249-255.

11. Rentrop KP, Cohen M, Blanke H, Phillips RA. Changes in collateral channel filling immediately after controlled coronary artery occlusion by an angioplasty balloon in human subjects. Journal of the American College of Cardiology 1985; 5 (3): 587-592.

12. Kilian JG, Keech A, Adams MR, Celermajer DS. Coronary collateralization: determinants of adequate distal vessel filling after arterial occlusion. Coronary artery disease 2002; 13 (3): 155-159.

13. Mayer M. Association of serum bilirubin concentration with risk of coronary artery disease. Clin Chem 2000; 46 (11): 1723-1727.
14. Yamaguchi T, Terakado M, Horio F, Aoki K, Tanaka M, Nakajima H. Role of bilirubin as an antioxidant in an ischemia-reperfusion of rat liver and induction of heme oxygenase. Biochem Biophys Res Commun 1996; 223 (1): 129-135.

15. Schwertner HA, Jackson WG, Tolan G. Association of low serum concentration of bilirubin with increased risk of coronary artery disease. Clin Chem 1994; 40 (1): 18-23.

16. Madamanchi NR, Vendrov A, Runge MS. Oxidative stress and vascular disease. Arterioscler Thromb Vasc Biol 2005; 25 (1): 29-38.

17. Erdogan D, Gullu H, Yildirim $\mathbf{E}$ et al. Low serum bilirubin levels are independently and inversely related to impaired flow-mediated vasodilation and increased carotid intima-media thickness in both men and women. Atherosclerosis 2006; 184 (2): 431-437.

18. Vitek L, Novotny L, Sperl M, Holaj R, Spacil J. The inverse association of elevated serum bilirubin levels with subclinical carotid atherosclerosis. Cerebrovasc Dis 2006; 21 (5-6): 408-414.

19. Turfan M, Duran M, Poyraz F et al. Inverse relationship between serum total bilirubin levels and severity of disease in patients with stable coronary artery disease. Coronary artery disease 2013; 24 (1): 29-32.

20. Chang CC, Hsu CY, Huang PH et al. Association of Serum Bilirubin with SYNTAX Score and Future Cardiovascular Events in Patients Undergoing Coronary Intervention. Acta Cardiol Sin 2016; 32 (4): 412-419.

21. Akboga MK, Canpolat U, Sahinarslan A et al. Association of serum total bilirubin level with severity of coronary atherosclerosis is linked to systemic inflammation. Atherosclerosis 2015; 240 (1): 110-114.

22. Nathoe HM, Koerselman J, Buskens E et al. Determinants and prognostic significance of collaterals in patients undergoing coronary revascularization. The American journal of cardiology 2006; 98 (1): 31-35.

23. Seiler C, Stoller M, Pitt B, Meier P. The human coronary collateral circulation: development and clinical importance. European heart journal 2013; 34 (34): 2674-2682.

Received May 19, 2019. Accepted July 1, 2019. 